\title{
DNA Extraction Protocols for Thermo Scientific Kingfisher- Customization Made Simple
}

SP\&A Application Laboratory, Vantaa, Finland

\section{Abstract}

Nucleic acid extraction is a routine, yet often time-consuming procedure, therefore making it ideal for automation. Thermo Scientific ${ }^{\top M}$ KingFisher $^{\top \mathrm{M}}$ instruments employ magnetic-particle technology for biomolecule isolation. Together with magnetic-particle-based kits, the instruments comprise a convenient and reliable extraction system that permits the allocation of precious laboratory hours to the subsequent applications and data analysis.

Nucleic acid extraction begins with sample homogenization and cell lysis. The pretreatment of the specimens is often the most labor-intensive element in the entire workload. In this note, we describe how to modify standard extraction protocols, and how these slight modifications may affect DNA yields and total extraction time.

\section{Introduction}

Cells, tissues and whole blood are typical starting materials for DNA extraction in

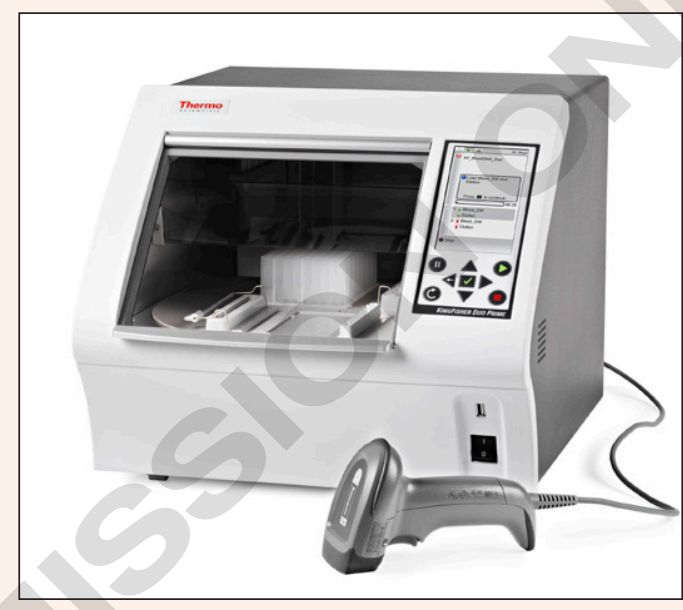

Figure 1. The KingFisher Duo Prime purification instrument

most molecular biology laboratories. Different facilities, however, may have different requirements for the extraction process. The important factors might be total time consumption, amount of hands-on work or purity or concentration of the obtained isolates. Typically, it is worthwhile to optimize the extraction protocols to meet the particular demands of a sample type or a downstream application. Although the optimization may initially require some effort, it saves a considerable amount of time and energy in the long run to avoid repetitive, unnecessary work.

The KingFisher instruments provide a semi-automated system that utilizes magnetic-particle technology for biomolecule isolation-including DNA, RNA, protein or cells. The instruments come with Thermo Scientific ${ }^{\mathrm{TM}}$ Bindlt ${ }^{\mathrm{TM}}$ software, which allows users to create novel protocols and modify existing extraction assays.

One common difficulty of DNA extraction is the sample pretreatment that includes cell lysis. The step is often performed manually prior to the actual DNA purification from the crude lysate. Though this practice aims to provide samples with the best possible DNA yield and purity, it also means more hands-on work for laboratory personnel. However, not all downstream experiments require vast quantities of exceptionally pure DNA. By pairing an optimized protocol with a particular sample type and application requirement, excessive workload can be easily reduced to a minimum.

In the following experiments, pre-existing Bindlt protocols for extracting DNA from cells and tissues or blood with the KingFisher Pure kits were modified with the software. With minor adjustments, it is possible to perform an internal lysis step on the instrument and otherwise compress the protocols to increase their walk-away capacity.

\section{Materials and Methods: \\ DNA Extraction from Cells}

Genomic DNA was extracted from $5 \times 10^{\wedge 5} \mathrm{HeLa}$-S3 cells on the KingFisher Duo Prime (\#5400110) with the KingFisher Cell and Tissue DNA Kit (\#97030196). The standard Cell and Tissue protocol was customized in three ways: Walk-Away, Dispense-1 and Dispense-2.

In the standard, unmodified protocol, the cell samples were pretreated manually in the laboratory. First, the cells were lysed with Proteinase K (hereafter ProtK) in the Lysis Buffer solution at $70^{\circ} \mathrm{C}$ for 15 minutes followed by RNase A treatment at room temperature for 5 minutes. The lysate was cleared with a brief centrifugation and the supernatants were transferred into a KingFisher deep-well 96 plate (\#95040450). The rest of the extraction was automated on the KingFisher Duo Prime. The running time of the protocol was 25 minutes.

The Walk-Away protocol eliminated almost all manual work as the lysis step was performed in the instrument

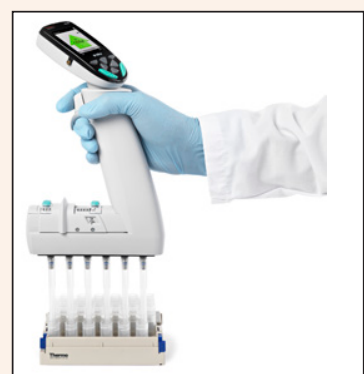

Figure 2. Sample pipetting using the Thermo Scientific ${ }^{\mathrm{TM}}$ E1-ClipTip ${ }^{\mathrm{TM}}$ Electronic Equalizer 6-channel, 15$1250 \mu$ pipette 
and all the necessary reagents were added to the deep-well plate at once. The protocol ran without interruptions for 39 minutes.

The two dispense protocols included either one or two pauses for supplementing pre-determined reagents to the plates. In the Dispense-1 protocol, the sample, Lysis Buffer with ProtK and RNase A were added to the deep-well plate prior to launching the run. After the lysis, the Magnetic Beads and Binding Buffer were pipetted to the sample wells and the protocol resumed. A more thorough dispense protocol contained two separate breaks, one for the addition of the RNase A and the other for the Magnetic Beads and Binding Buffer. The Dispense-1 protocol took 43 minutes to complete, whereas the Dispense-2 was 48 minutes long.

\section{DNA Extraction from Whole Blood}

Genomic DNA was extracted from $200 \mu$ of EDTA-treated whole blood with the KingFisher Pure DNA Blood Kit (\#98010196) on the KingFisher Duo Prime. In the unmodified protocol, the lysis was performed within the instrument and the Magnetic Beads and Binding Buffer were added to the lysed samples during a pause. The newly created walk-away protocol, on the other hand, did not contain any breaks, and instead, all the reagents were added to the deep-well plate before commencing the run. The standard and the walk-away protocols took 33 and 38 minutes to complete, respectively (Figure 3).

Reagents and samples were pipetted with the Thermo Scientific ${ }^{\mathrm{TM}}$ E1-ClipTip ${ }^{\mathrm{TM}}$ Electronic Equalizer 6-channel, 15-1250 $\mu$ pipette (\#4672090), which features adjustable tip spacing to transfer samples from tubes to plates. This saves hands-on time, and reduces repetitive motions required to add samples, reagents and magnetic beads on the deep-well 96-well plate or 12-well elution strip-while using one pipette.
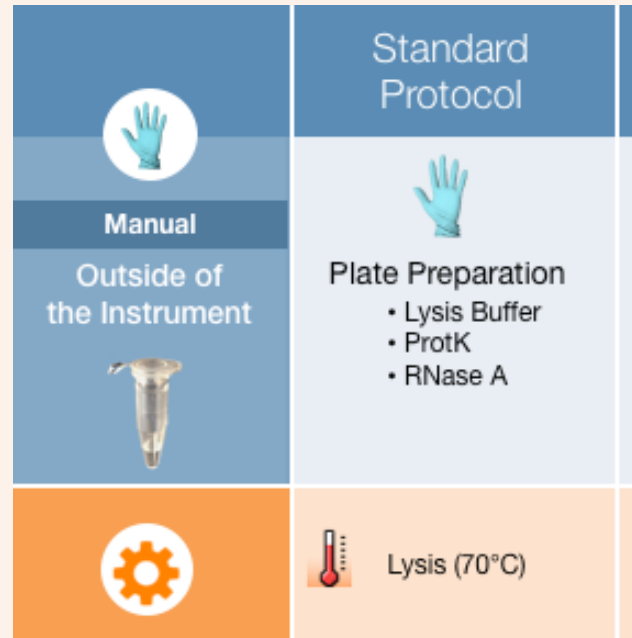

\section{Plate Preparation \\ - Lysis Buffer \\ - ProtK \\ - RNase A}

Automated

Protocal in

Instrument
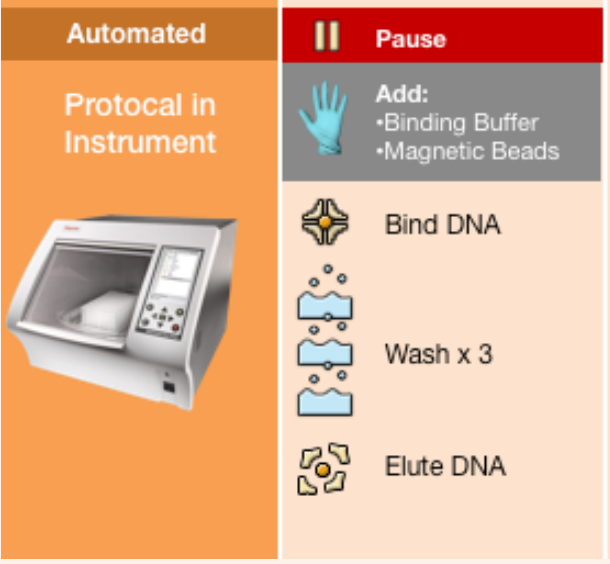

Lysis $\left(70^{\circ} \mathrm{C}\right)$
Walk-Away

Protocol

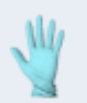

Plate Preparation

- Lysis Buffer

- ProtK

- RNase A

- Binding Buffer

- Magnetic Beads

Lysis $\left(70^{\circ} \mathrm{C}\right)$

\section{Bind DNA}

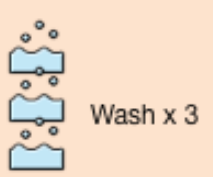

Figure 3. Genomic DNA extraction process from EDTA-treated whole blood using the KingFisher Pure DNA Blood Kit

\section{Results}

The results demonstrate that the cell lysis can be carried out within the instrument and many of the manual steps can be automated on the KingFisher instrument (Figures 4 and 5). The slight modifications described above radically shorten or even eliminate most of the manual work of the extraction procedures. Though the alterations may affect the total yield of the obtained DNA, the isolates are of high quality and suitable for multiple downstream applications such as various enzymatic assays. Similar protocol adaptations can be easily applied to other sample materials, such as viral particles, to facilitate nucleic acid extraction.

The Bindlt software can be used to customize the existing protocols to meet the quality and yield expectations. Choosing the most suitable protocols saves time and effort in the long run.

\section{Summary}

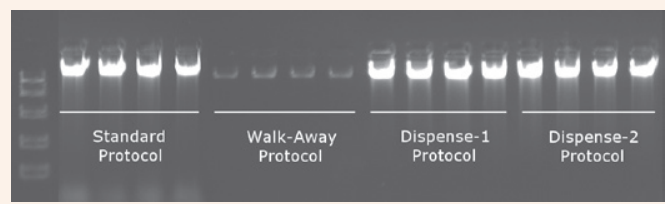

Figure 4. Genomic DNA purified from cultured HeLa-S3 cells with four different protocols

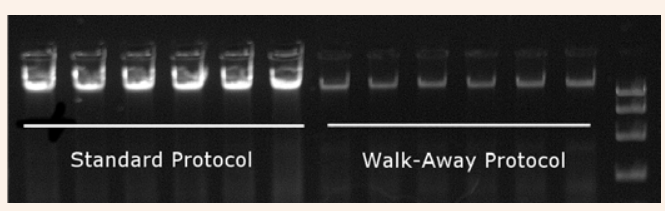

Figure 5. Genomic DNA purified from whole blood with the standard and Walk-Away protocols

The KingFisher instruments provide a versatile, automated sample processing sys-

tem for routine extraction of DNA, RNA, protein or cells. When it comes to efficient sample purification, the KingFisher instruments are like a reliable extra set of hands in the laboratory. With minor modification to the standard DNA extraction protocols, manual work can be cut down to the bare minimum, all the while retaining a satisfactory DNA quality and yield. 